\title{
Model-Based Development and Implementation of Real-Time Object Spatial Attitude Estimation
}

\author{
Bui Hong Hue, Tran Xuan Kien, Doan Minh Dinh, and Do Duc Hanh
}

\begin{abstract}
This paper presents the development and implementation of a low cost attitude estimation module where an extended Kalman filter is used for real time processing of signals obtained from an inertial Micro-Electro-Mechanical System (MEMS) sensor module to estimate the attitude of an object in space. MATLAB/Simulink Embedded tools are used to design, compile, and download directly into the target. An update rate of $100 \mathrm{~Hz}$ can be achieved using a Microchip 16 bit dsPIC microcontroller.
\end{abstract}

Index Terms-Attitude estimation, embedded systems, extended kalman filter, MEMS sensors.

\section{INTRODUCTION}

Unmanned Aerial Vehicles (UAVs) are a rapidly growing area of research and development. One of the biggest technical barriers is attitude estimation. Attitude estimation [1] includes estimating vehicle's orientation from body measurements and known reference observations, and filtering of noisy measurements. Attitude estimations should be as accurate as possible. The accuracy of an estimator is affected by the noises from its sensors and platform. Vibration of the vehicle also makes some noise. These noises need to be filtered out. Typically gyroscopes can be used to calculate the attitude of the vehicle by integrating their signals. Nowadays low cost attitude estimation modules could be developed using cheap Micro-Electro-Mechanical System (MEMS) inertial sensors combined with high speed digital signal processors (DSPs) or microcontroller with digital signal processing support [2]-[4]. However MEMS sensors have more noise and bias drifts that need to be eliminated since the accumulation of them could make a reasonable error over time. The design of such attitude estimation requires either an expensive commercial module or significant knowledge of electronics, attitude dynamics, and Kalman filtering [5], [6]. Quaternions are especially appealing since no singularities are present and the kinematics equation is bilinear. This paper presents in detail the development and implementation of a low cost attitude estimation module where an extended Kalman filter with full quaternion is applied as a solution to the complementary sensor fusion problem for attitude estimation on a Microchip

Manuscript received February 1, 2013; revised March 15, 2013.

B. H. Hue is with the College of Urban Works Construction, Hanoi, Vietnam (e-mail: ngochuy.buihue@gmail.com).

T. X. Kien is with the Military Science and Technology Institute, Hanoi, Vietnam (e-mail: txkien2003@yahoo.com).

D. M. Dinh is with the Department of Science and Technology, Ministry of Defense, Vietnam (email: doanminhdinh@vnn.vn).

D. D. Hanh is with the Department of Control Engineering, Military Technical Academy, Hanoi, Vietnam (email: hanh@aitcv.ac.vn). 16-bit dsPIC microcontroller.

The rest of the paper is organized as follows. In Section II, the basic concepts of system state estimation, extended Kalman filter and attitude dynamics are presented. The attitude estimation algorithm, hardware and software implementation is introduced in Section III. The results of performance testing are given in Section IV. We conclude in Section V.

\section{State Estimation And EXTEnded KaLMAn FiLter}

\section{A. State Estimation}

An ideal discrete dynamic system is modeled by the following difference equation [3]:

$$
\vec{x}_{k}=f_{k-1}\left(\vec{x}_{k-1}\right)
$$

where $\vec{x}$ is known as the state of the system. In a physical system there are disturbances and hence it is more accurate to include a system noise term $\vec{\omega}$, i.e.

$$
\begin{aligned}
& \vec{x}_{k}=f_{k-1}\left(\vec{x}_{k-1}\right)+\vec{\omega}_{k-1} \\
& \vec{\omega}_{k} \sim N\left(0, Q_{k}\right)
\end{aligned}
$$

The second line means that $\vec{\omega}_{k}$ is randomly distributed with a Gaussian probability distribution of covariance $Q_{k}$.

In general, the values of $x_{k}$ are not directly accessed and obtained through measurements which are inherently noisy and modeled by the following equation:

$$
\begin{aligned}
& \vec{z}_{k}=h_{k}\left(\vec{x}_{k}\right)+\vec{v}_{k} \\
& \vec{v}_{k} \sim N\left(0, R_{k}\right)
\end{aligned}
$$
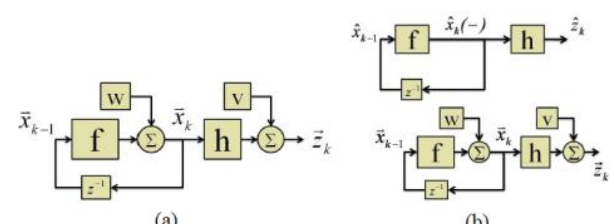

(b)
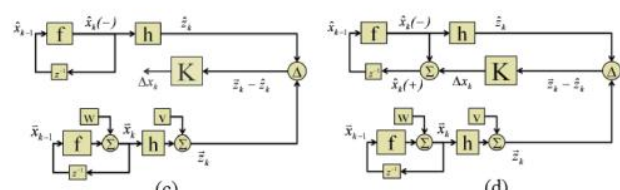

(d)

Fig. 1. System dynamics, observer and state estimation

The complete system is represented by a diagram in Fig. 1(a) where $\mathrm{z}^{-1}$ is the unit delay operator. To estimate the state $\vec{x}_{k}$, an observer is introduced. The observer has virtual state 
$\hat{x}_{k}$, virtual measurements $\hat{z}_{k}$, and obeys the same equations as the real system but without noises, i.e.

$$
\begin{aligned}
& \hat{x}_{k}=f_{k-1}\left(\hat{x}_{k-1}\right) \\
& \hat{z}_{k}=h_{k}\left(\hat{x}_{k}\right)
\end{aligned}
$$

The observer is assumed to run in parallel with the real system (see Fig. 1(b)). Because of the system noise $\vec{\omega}$, the $a$ priori propagated state $\hat{x}_{k}(-)$ differs from the true state $\vec{x}_{k}$ and virtual measurement $\hat{z}_{k}$ does not agree with the actual measurement $\vec{z}_{k}$. Feedback gain $K_{k}$ is introduced to estimate the state error $\Delta \hat{x}_{k}=\vec{x}_{k}-\hat{x}_{k}$ based on the measurement error $\vec{z}_{k}-\hat{z}_{k}$ (see Fig. 1(c)). Finally, this error is used to correct the observer's state, forming the a posteriori corrected state $\hat{x}_{k}(+)$ (see Fig. 1(d)).

\section{B. Extended Kalman Filter}

The expectation value of the state error $\Delta \hat{x}_{k}$ is known as the covariance of the states expressed by the following equation:

$$
P_{k}=\varepsilon\left(\left[\vec{x}_{k}-\hat{x}_{k}\right]\left[\vec{x}_{k}-\hat{x}_{k}\right]^{T}\right)
$$

An optimal feedback gain $K_{k}$ can be derived by minimizing $P_{k}$. For linear systems this is known as the Kalman filter and was first presented in 1960 by Kalman [6]. The estimated state and covariance are propagated at every time step, but in many systems measurements are only sporadically available. In this case the state covariance grows for many time steps as the estimated state diverges from the true state. When a measurement becomes available, the feedback gain $K$ is calculated, the state is corrected, and the state covariance is updated (reduced) accordingly.

The Kalman filter has been applied to nonlinear systems in many different ways. One of the most straightforward techniques is to linearize the system dynamics and the measurement function around the expected state $\vec{x}_{k}(-)$, and then apply the Kalman filter as normal. This is known as extended Kalman filtering and the implementation equations (borrowed from [5]) are presented in Table 1.

\begin{tabular}{l|cc|}
\multicolumn{1}{c|}{ TABLE I: EXTENDED KALMAN FILTER IMPLEMENTATION EQUATIONS } \\
\hline \hline $\begin{array}{l}\text { System } \\
\text { dynamics }\end{array}$ & $\begin{array}{r}\vec{x}_{k}=f_{k-1}\left(\vec{x}_{k-1}\right)+\vec{\omega}_{k-1} \\
\vec{\omega}_{k} \sim N\left(0, Q_{k}\right)\end{array}$ & $(6.1)$ \\
\hline Measurement & $\vec{z}_{k}=h_{k}\left(\vec{x}_{k}\right)+\vec{v}_{k}$ & $(6.3)$ \\
$\vec{v}_{k} \sim N\left(0, R_{k}\right)$ & $(6.4)$ \\
\hline Covariance & $P_{k}=\varepsilon\left(\left[\vec{x}_{k}-\hat{x}_{k}\right]\left[\vec{x}_{k}-\hat{x}_{k}\right]^{T}\right)$ & $(6.5)$ \\
\hline $\begin{array}{l}\text { State } \\
\text { propagation }\end{array}$ & $\hat{x}_{k}(-)=\Phi_{k-1}\left(\hat{x}_{k-1}(+)\right)$ & $(6.6)$ \\
\hline $\begin{array}{l}\text { Dynamics } \\
\text { linearization }\end{array}$ & $\Phi_{k-1}=\left.\frac{\partial f_{k-1}}{\partial x}\right|_{x=\hat{x}_{k-1}(+)}$ \\
\hline $\begin{array}{l}\text { Predicted } \\
\text { measurement }\end{array}$ & $\vec{z}_{k}=h_{k} \hat{x}_{k}(-)$ \\
\hline $\begin{array}{l}\text { Measurement } \\
\text { linearization }\end{array}$ & $H_{k}=\left.\frac{\partial h_{k}}{\partial x}\right|_{x=\hat{x}_{k}(-)}$ \\
\hline
\end{tabular}

\begin{tabular}{|l|c|}
\hline \hline $\begin{array}{l}\text { State covariance } \\
\text { propagation }\end{array}$ & $P_{k}(-)=\Phi_{k-1} P_{k-1}(+) \Phi_{k-1}^{T}+Q_{k-1}$ (6.10) \\
\hline Feedback gain & $K_{k}=P_{k}(-) H_{k}^{T} H_{k} P_{k}(-) H_{k}^{T}+R_{k} \quad(6.11)$ \\
\hline State update & $\hat{x}_{k}(+)=\hat{x}_{k}(-)+K_{k}\left(z_{k}-\hat{z}_{k}\right)$ \\
\hline $\begin{array}{l}\text { State covariance } \\
\text { update }\end{array}$ & $P_{k}(+)=\left(1-K_{k} H_{k}\right) P_{k}(-)$ \\
\hline \hline
\end{tabular}

Extended Kalman filter allows to dynamically estimate attitude and drifting gyro biases, as long as they are modeled as states in our dynamical system. Next the three-dimensional attitude dynamics and measurement equations will be derived.

\section{Attitude Dynamics}

Suppose there is a rigid body with the body coordinate system OXYZ and let $\mathrm{O}_{0} \mathrm{X}_{0} \mathrm{Y}_{0} \mathrm{Z}_{0}$ be a reference (e.g. earth) coordinate system (Fig. 2). Translate the origin of the reference coordinate system to the origin of the body coordinate system (keeping the directions of the axes $\mathrm{O}_{0} \mathrm{X}_{0}$, $\mathrm{O}_{0} \mathrm{Y}_{0}$ and $\mathrm{O}_{0} \mathrm{Z}_{0}$ unchanged). The angles representing the relationship between these two coordinate systems are called orientation angles (attitude Euler angles) including yaw angle $\psi$, pitch angle $\theta$ and roll angle $\varphi$.

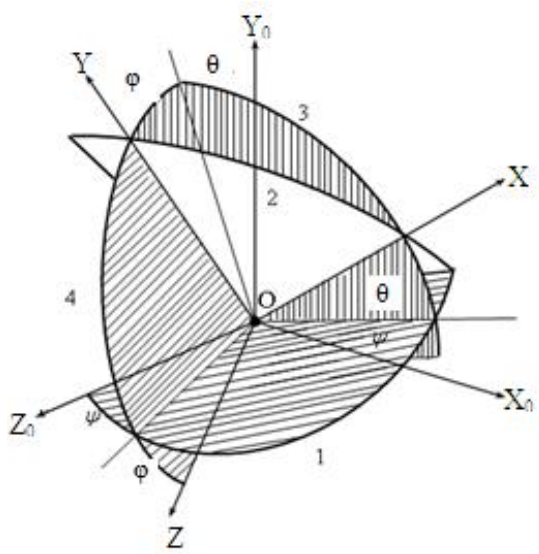

Fig. 2. Rigid body coordinate system and reference coordinate system. 1 The horizontal plane; 2 - The object symetry plane; 3 - The vertical plane containing the longitudinal axis; 4 - The vertical plane containing OZ axis.

From orientation angles, direction cosine matrix (DCM) between two coordinate systems is calculated as follows [3]:

$$
\begin{aligned}
& \left(\begin{array}{l}
u_{b} \\
v_{b} \\
w_{b}
\end{array}\right)=T(\varphi, \theta, \psi)\left(\begin{array}{l}
u_{e} \\
v_{e} \\
w_{e}
\end{array}\right) \\
& T=\left[\begin{array}{ccc}
\cos \psi \cos \theta & \sin \theta & \sin \psi \cos \theta \\
-\cos \psi \sin \theta \cos \varphi+\sin \psi \sin \varphi & \cos \theta \cos \varphi & \sin \psi \sin \theta \cos \varphi+\cos \psi \sin \varphi \\
\cos \psi \sin \theta \sin \varphi+\sin \psi \cos \varphi & -\cos \theta \sin \varphi \sin \psi \sin \theta \sin \varphi+\cos \psi \cos \varphi
\end{array}\right]
\end{aligned}
$$

where $\left(u_{e} ; v_{e} ; w_{e}\right)$ is a vector in the reference frame, $\left(u_{b} ; v_{b} ; w_{b}\right)$ is the vector in the rigid body frame $(\mathrm{X} ; Y ; Z)$.

It turns out that the Euler angle dynamics can be derived by inspection alone. Because the body rotation rates $\vec{\omega}=\left[\begin{array}{lll}\omega_{x} & \omega_{y} & \omega_{z}\end{array}\right]$ are defined as instantaneous rotation rates about the $O X, O Y$, and $O Z$ axes respectively, it can be observed that $\phi$ is a rotation about the $O X$ axis. $\theta$ is a rotation about an intermediate axis, so to project $\dot{\theta}$ onto the body frame it must be rotated using the $\phi$ rotation. Likewise, 
$\dot{\psi}$ must be rotated about first $\theta$, and then $\phi$, i.e.

$$
\left(\begin{array}{c}
\omega_{x} \\
\omega_{y} \\
\omega_{z}
\end{array}\right)=\left(\begin{array}{c}
\dot{\phi} \\
0 \\
0
\end{array}\right)+T_{\phi}\left(\begin{array}{c}
0 \\
\dot{\theta} \\
0
\end{array}\right)+T_{\phi} T_{\theta}\left(\begin{array}{c}
0 \\
0 \\
\dot{\psi}
\end{array}\right)
$$

From (8) and (7) we obtain:

$$
\left(\begin{array}{c}
\dot{\phi} \\
\dot{\theta} \\
\dot{\psi}
\end{array}\right)=\left(\begin{array}{ccc}
1 & \sin (\phi) \tan (\theta) & \cos (\phi) \tan (\theta) \\
0 & \cos (\phi) & -\sin (\phi) \\
0 & \sin (\phi) \sec (\theta) & \cos (\phi) \sec (\theta)
\end{array}\right)\left(\begin{array}{l}
\omega_{x} \\
\omega_{y} \\
\omega_{z}
\end{array}\right)
$$

Quaternions are hyper-complex numbers of rank 4. A quaternion $q$ can be expressed as:

$$
q=q_{0}+i q_{1}+j q_{2}+k q_{3}
$$

Then the DCM is a function of $q$ :

$$
\begin{aligned}
& \left(\begin{array}{l}
u_{b} \\
v_{b} \\
w_{b}
\end{array}\right)=T(q)\left(\begin{array}{l}
u_{e} \\
v_{e} \\
w_{e}
\end{array}\right) \\
& T=\left(\begin{array}{ccc}
2 q_{0}^{2}+2 q_{1}^{2}-1 & 2 q_{1} q_{2}+2 q_{0} q_{3} & 2 q_{1} q_{3}-2 q_{0} q_{2} \\
2 q_{1} q_{2}-2 q_{0} q_{3} & 2 q_{0}^{2}+2 q_{2}^{2}-1 & 2 q_{2} q_{3}+2 q_{0} q_{1} \\
2 q_{1} q_{3}+2 q_{0} q_{2} & 2 q_{2} q_{3}-2 q_{0} q_{1} & 2 q_{0}^{2}+2 q_{3}^{2}-1
\end{array}\right)
\end{aligned}
$$

\section{IMPLEMENTATION}

\section{A. Implementation Equations}

The attitude estimation module is developed using an extended Kalman filter with the full quaternion $[q 0$ q1 $q 2$ $q 3]^{T}$. The state is $\vec{x}=\left[q_{0} q_{1} q_{2} q_{3} \omega_{1} \omega_{2} \omega_{3} b_{1} b_{2} b_{3}\right]^{T}$, where $b_{i}$ are the gyro biases. The implementation follows equations summarized in Table 1 and it is necessary to derive the state transfer functions $f$ and $\Phi$, the measurement functions $\mathrm{h}$ and $\mathrm{H}$, and the noise covariances $Q$ and $R$.

\section{1) State transfer functions}

Rotation angular velocities and drifts of the gyro are assumed to be stochastic processes, i.e.

$$
\begin{aligned}
& \vec{\omega}_{k}=\vec{\omega}_{k-1}+\vec{\omega}_{\omega, k-1} \\
& \vec{b}_{k}=\vec{b}_{k-1}+\vec{\omega}_{b, k-1}
\end{aligned}
$$

State transfer function $f$ is derived from the above equation and equation 6.1. From equation $6.7, \Phi$ is obtained by taking partial derivatives of $f$.

The state $\vec{x}_{k+1}$ is represented as follows:

$$
\left(\begin{array}{cc}
q & 0, k+1 \\
q & 1, k+1 \\
q & 2, k+1 \\
q & 3, k+1 \\
\omega & 1, k+1 \\
\omega & 2, k+1 \\
\omega & 3, k+1 \\
b & 1, k+1 \\
b & 2, k+1 \\
b & 3, k+1
\end{array}\right)=\left(\begin{array}{c}
q 0, k \\
q 1, k \\
q 22, k \\
q 3, k
\end{array}\right)
$$

$$
\Pi=\left(\begin{array}{cccc}
\cos \left[\frac{|\omega \cdot \Delta t|}{2}\right] & -\frac{\omega_{1}}{|\omega|} \sin \left[\frac{|\omega| \cdot \Delta t}{2}\right] & -\frac{\omega_{2}}{|\omega|} \sin \left[\frac{|\omega| \cdot \Delta t}{2}\right] & -\frac{\omega_{3}}{|\omega|} \sin \left[\frac{|\omega| \cdot \Delta t}{2}\right] \\
\frac{\omega_{1}}{|\omega|} \sin \left[\frac{|\omega| \cdot \Delta t}{2}\right] & \cos \left[\frac{|\omega \cdot \Delta t|}{2}\right] & \frac{\omega_{3}}{|\omega|} \sin \left[\frac{|\omega| \cdot \Delta t}{2}\right] & -\frac{\omega_{2}}{|\omega|} \sin \left[\frac{|\omega| \cdot \Delta t}{2}\right] \\
\frac{\omega_{2}}{|\omega|} \sin \left[\frac{|\omega| \cdot \Delta t}{2}\right] & -\frac{\omega_{3}}{|\omega|} \sin \left[\frac{|\omega| \cdot \Delta t}{2}\right] & \cos \left[\frac{|\omega \cdot \Delta t|}{2}\right] & \frac{\omega_{1}}{|\omega|} \sin \left[\frac{|\omega| \cdot \Delta t}{2}\right] \\
\frac{\omega_{3}}{|\omega|} \sin \left[\frac{|\omega| \cdot \Delta t}{2}\right] & \frac{\omega_{2}}{|\omega|} \sin \left[\frac{|\omega| \cdot \Delta t}{2}\right] & -\frac{\omega_{1}}{|\omega|} \sin \left[\frac{|\omega| \cdot \Delta t}{2}\right] & \cos \left[\frac{|\omega \cdot \Delta t|}{2}\right]
\end{array}\right)
$$

\section{2) Measurement functions}

Measurement of gravity and inertial acceleration in the body frame is expressed by:

$$
\vec{z}_{\text {accel }}=T\left(\begin{array}{l}
\ddot{x} \\
\ddot{y} \\
\ddot{z}-g
\end{array}\right)
$$

Most efficient computation is with aacurate tracking estimation, i.e. $\hat{q} \approx q$, and then subtracting the inertial accelerations measured by GPS we obtain:

$$
z_{\text {accel }}=T_{q}\left(\begin{array}{c}
\ddot{x} \\
\ddot{y} \\
\ddot{z}-g
\end{array}\right)-T_{\hat{q}}\left(\begin{array}{c}
\ddot{x} \\
\ddot{y} \\
\ddot{z}
\end{array}\right) \approx T_{q}\left(\begin{array}{c}
0 \\
0 \\
-g
\end{array}\right)
$$

After coordinate transformation we obtain:

$z_{\text {aceel }}=\left(\begin{array}{lll}2 q_{0}^{2}+2 q_{1}^{2}-1 & 2 q_{1} q_{2}+2 q_{0} q_{3} & 2 q_{1} q_{3}-2 q_{0} q_{2} \\ 2 q_{1} q_{2}-2 q_{0} q_{3} & 2 q_{0}^{2}+2 q_{2}^{2}-1 & 2 q_{2} q_{3}+2 q_{0} q_{1} \\ 2 q_{1} q_{3}+2 q_{0} q_{2} & 2 q_{2} q_{3}-2 q_{0} q_{1} & 2 q_{0}^{2}+2 q_{3}^{2}-1\end{array}\right)\left(\begin{array}{c}0 \\ 0 \\ -g\end{array}\right)=\left(\begin{array}{c}-2 g\left(q_{1} q_{3}+q_{0} q_{2}\right) \\ -2 g\left(q_{2} q_{3}-q_{0} q_{1}\right) \\ -g\left(2 q_{0}^{2}+2 q_{3}^{2}-1\right)\end{array}\right)$

Local (Earth) magnetic field is measured, given as

$$
z_{\text {mag }}=\left(\begin{array}{ccc}
2 q_{0}^{2}+2 q_{1}^{2}-1 & 2 q_{1} q_{2}+2 q_{0} q_{3} & 2 q_{1} q_{3}-2 q_{0} q_{2} \\
2 q_{1} q_{2}-2 q_{0} q_{3} & 2 q_{0}^{2}+2 q_{2}^{2}-1 & 2 q_{2} q_{3}+2 q_{0} q_{1} \\
2 q_{1} q_{3}+2 q_{0} q_{2} & 2 q_{2} q_{3}-2 q_{0} q_{1} & 2 q_{0}^{2}+2 q_{3}^{2}-1
\end{array}\right)\left(\begin{array}{l}
B_{x} \\
B_{y} \\
B_{z}
\end{array}\right)
$$

Measured body's angular velocities including drifts are given by:

$$
\vec{z}_{\omega}=\left(\begin{array}{l}
\omega_{1}+b_{1} \\
\omega_{2}+b_{2} \\
\omega_{3}+b_{3}
\end{array}\right)
$$

Combining equations (14), (15), and (16), the full measurement function $h$ is derived (by 6.8). From equation 6.9 , the linearized measurement function $\mathrm{H}$ is obtained by taking partial derivatives of $h$, given as

$$
H=\left(\begin{array}{ccc}
2 g q_{2} & -2 g q_{3} & 2 g q_{0} \\
-2 g q_{1} & -2 g q_{0} & -2 g q_{3} \\
-4 g q_{0} & 0 & 0 \\
4 B_{x} q_{0}-2 B_{z} q_{2}+2 B_{y} q_{3} & 4 B_{x} q_{1}+2 B_{y} q_{2}+2 B_{z} q_{3} & -2 B_{z} q_{0}+2 B_{y} q_{1} \\
4 B_{y} q_{0}+2 B_{z} q_{1}-2 B_{x} q_{3} & 2 B_{z} q_{0}+2 B_{x} q_{2} & 4 B_{y} q_{0}+2 B_{z} q_{1}-2 B_{x} q_{3} \\
4 B_{z} q_{0}-2 B_{y} q_{1}+2 B_{x} q_{2} & 2 B_{z} q_{0}+2 B_{x} q_{2} & 2 B_{x} q_{0}+2 B_{y} q_{3} \\
0 & -2 B_{y} q_{0}+2 B_{x} q_{3} & 0 \\
0 & 0 & 0 \\
0 & 0 & 0
\end{array}\right.
$$




$$
\left.\begin{array}{ccccccc}
-2 g q_{1} & 0 & 0 & 0 & 0 & 0 & 0 \\
-2 g q_{2} & 0 & 0 & 0 & 0 & 0 & 0 \\
-4 g q_{3} & 0 & 0 & 0 & 0 & 0 & 0 \\
2 B_{y} q_{0}+2 B_{z} q_{1} & 0 & 0 & 0 & 0 & 0 & 0 \\
-2 B_{x} q_{0}+2 B_{z} q_{2} & 0 & 0 & 0 & 0 & 0 & 0 \\
4 B_{y} q_{0}+2 B_{z} q_{1}-2 B_{x} q_{3} & 0 & 0 & 0 & 0 & 0 & 0 \\
0 & 1 & 0 & 0 & 1 & 0 & 0 \\
0 & 0 & 1 & 0 & 0 & 1 & 0 \\
0 & 0 & 0 & 1 & 0 & 0 & 1
\end{array}\right)
$$

\section{3) System and measurement noise covariances}

The system noise covariance is assumed to be:

$$
Q=\left(\begin{array}{cccccccccc}
\sigma_{q}^{2} & 0 & 0 & 0 & 0 & 0 & 0 & 0 & 0 & 0 \\
0 & \sigma_{q}^{2} & 0 & 0 & 0 & 0 & 0 & 0 & 0 & 0 \\
0 & 0 & \sigma_{q}^{2} & 0 & 0 & 0 & 0 & 0 & 0 & 0 \\
0 & 0 & 0 & \sigma_{q}^{2} & 0 & 0 & 0 & 0 & 0 & 0 \\
0 & 0 & 0 & 0 & \sigma_{\omega}^{2} & 0 & 0 & 0 & 0 & 0 \\
0 & 0 & 0 & 0 & 0 & \sigma_{\omega}^{2} & 0 & 0 & 0 & 0 \\
0 & 0 & 0 & 0 & 0 & 0 & \sigma_{\omega}^{2} & 0 & 0 & 0 \\
0 & 0 & 0 & 0 & 0 & 0 & 0 & \sigma_{b}^{2} & 0 & 0 \\
0 & 0 & 0 & 0 & 0 & 0 & 0 & 0 & \sigma_{b}^{2} & 0 \\
0 & 0 & 0 & 0 & 0 & 0 & 0 & 0 & 0 & \sigma_{b}^{2}
\end{array}\right)
$$

where $\sigma_{\mathrm{q}}$ is the expected quaternion system noise, $\sigma_{\omega}$ is the random walk on the body rates, and $\sigma_{\mathrm{b}}$ is the random walk on the gyro biases.

The sensor or measurement noise covariance is given as:

$$
R=\left(\begin{array}{ccccccccc}
\sigma_{a}^{2} & 0 & 0 & 0 & 0 & 0 & 0 & 0 & 0 \\
0 & \sigma_{a}^{2} & 0 & 0 & 0 & 0 & 0 & 0 & 0 \\
0 & 0 & \sigma_{a}^{2} & 0 & 0 & 0 & 0 & 0 & 0 \\
0 & 0 & 0 & \sigma_{\text {mag }}^{2} & 0 & 0 & 0 & 0 & 0 \\
0 & 0 & 0 & 0 & \sigma_{\text {mag }}^{2} & 0 & 0 & 0 & 0 \\
0 & 0 & 0 & 0 & 0 & \sigma_{m a g}^{2} & 0 & 0 & 0 \\
0 & 0 & 0 & 0 & 0 & 0 & \sigma_{\omega}^{2} & 0 & 0 \\
0 & 0 & 0 & 0 & 0 & 0 & 0 & \sigma_{\omega}^{2} & 0 \\
0 & 0 & 0 & 0 & 0 & 0 & 0 & 0 & \sigma_{\omega}^{2}
\end{array}\right)
$$

where, $\sigma_{a}, \sigma_{m a g}$ and $\sigma_{\omega}$ are the standard deviations of the expected noises of the accelerometers, magnetometers, and rate gyros respectively.

This implementation is computationally simpler than an Euler angle implementation, but it is still too intensive to run in floating point on a 16-bit microcontroller at a reasonable update rate.

\section{B. Hardware Design}

The attitude estimation module contains a dsPIC33f-based board, auxiliary blocks including power supply, blocks for communication and interface with MEMS sensors (using I2C standard), GPS (COM1) and data transmission of data to PC (COM2). The MEMS sensor block (of GY85 type) has sensors for angular velocity, acceleration and magnetic field (magnetometer). The GPS block is of u-blox 5S type.

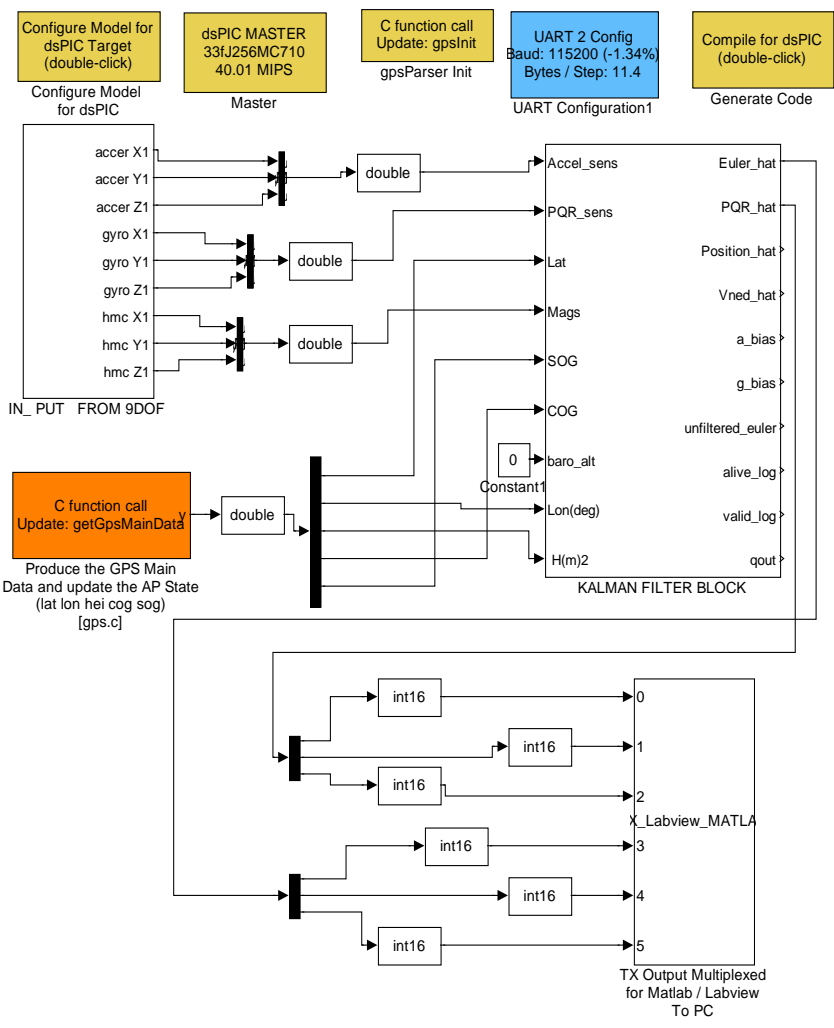

Fig. 3. Simulink implementation of the designed attitude estimation.

\section{Software Design}

Development and implementation of algorithm for attitude estimation on a high speed dsPIC microcontroller are done using compile chain tools from Mathworks and Microchip instead of procedural $(\mathrm{C} / \mathrm{C}++)$ programming. These tools allow convenient and fast design of computation and processing models with graphical design, drag and drop of blocks from Simulink built-in libraries, corresponding blocks for dsPIC available in MATLAB/Simulink and MATLAB functions ( $\mathrm{m}$. file, s-function). Simulink modules for simulation and generation of embedded application programs can be compiled and then downloaded directly into the dsPIC microcontroller target. Modules for attitude estimation, gyro drift (biases), quaternions are designed as shown in Fig. 3.

The main module contains a sub-module for reading data from MEMS sensors through I2C interface, function for reading GPS data from COM1, sub-module for Kalman filter, sub-module for sending output data to PC through COM2.

After compiling with Real-time Workshop Embedded Coder the code is downloaded into the target using a Microchip Development Programmer/Debugger PICKit 2.

\section{Performance Testing}

To test the implemented attitude estimation module for performance, the module is mounted on a 1573 Series Automatic Positioning and Rate Table System (the Test Table System) from the Ideal Aerosmith as shown in Fig. 4. The Test Table System is designed to provide precise and reliable position, rate and acceleration motion control for the development and/or production testing of inertial packages and their components.

The Test Table System can be operated from the AERO 
832 Controller front panel for local control or through a computer interface for remote control and is programmed with the Ideal Aerosmith Table Language (ATL) for remote operation.

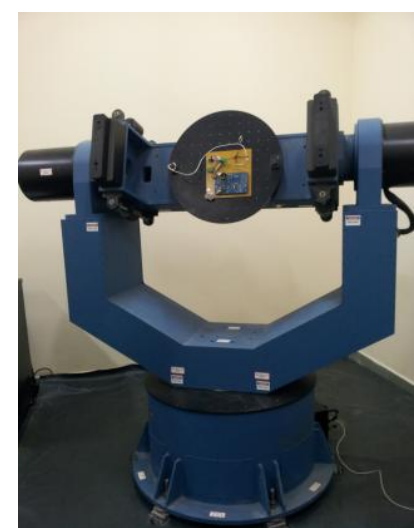

Fig. 4. 1573 Series multi-axis precision positioning and rate Table System

The Test Table System can provide position, rate and acceleration motion with position accuracy of \pm 20 arc sec, rate accuracy of $0.005 \%$, and simulate trapezoidal velocity profiles with programmable velocity and acceleration, sinusoidal motion profiles with programmable amplitude and frequency and complex motion profiles.

Fig. 5 shows the graph of estimation angles and angular velocities about $\mathrm{Y}$ axis when the module is mounted in the Test Table System that rotates about $\mathrm{Y}$ axis from $0^{\circ}$ to $60^{\circ}$ with angular velocity of $10^{\circ}$ s (as shown in Line 1 ). Lines 2 and 3 show estimation angles and angular velocities about $\mathrm{Y}$ axis respectively.

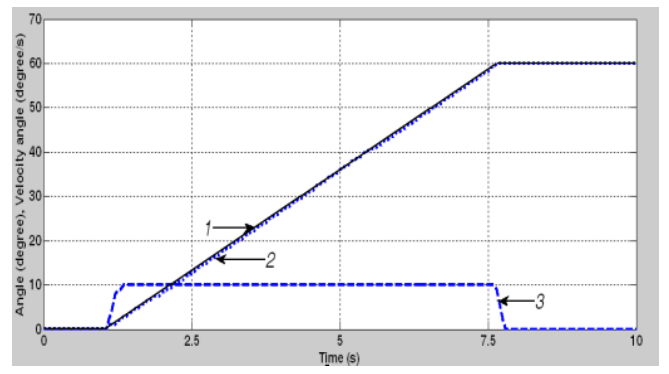

Fig. 5. Experiment results when rotating the object in space; 1- Rotation angle of the Test Table System about Y axis; 2 - estimation angles about $Y$ axis; 3 - estimation angular velocities about $\mathrm{Y}$ axis.

The graph in Fig. 6 shows estimation angles (dashed line 2) and angular velocities (dashed line 3 ) obtained from the implemented module when the Test Table System rotates (with sinusoidal motion profile) about $\mathrm{Z}$ axis from 0 to $180^{\circ}$ with rate of $240 \%$ s (solid line 1 ).

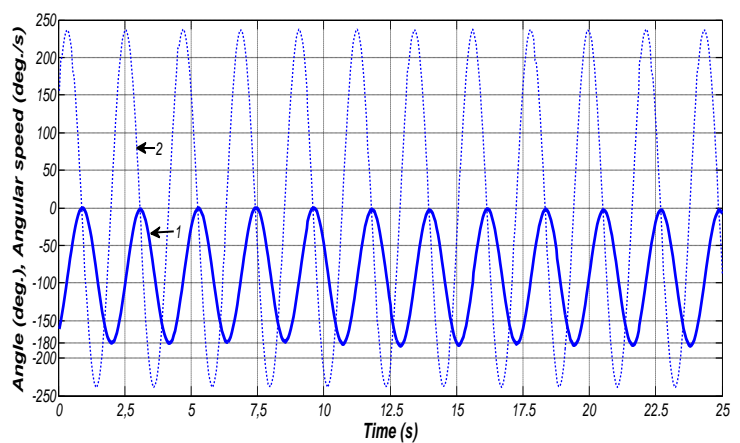

Fig. 6. Estimation angle and angular velocity graphs when the Test Table System rotates (with sinusoidal motion profile) about $\mathrm{Z}$ axis from 0 to $180^{\circ}$ with rate of $240^{\circ}$ s.
Experiment results in Fig. 7 show estimation angles and angular velocities when the module (not mounted in the Test Table System) is rotating $90^{\circ}$ clockwise and then counter-clockwise about $\mathrm{X}$ axis in $4^{\text {th }}$ and $12^{\text {th }}$ seconds respectively.

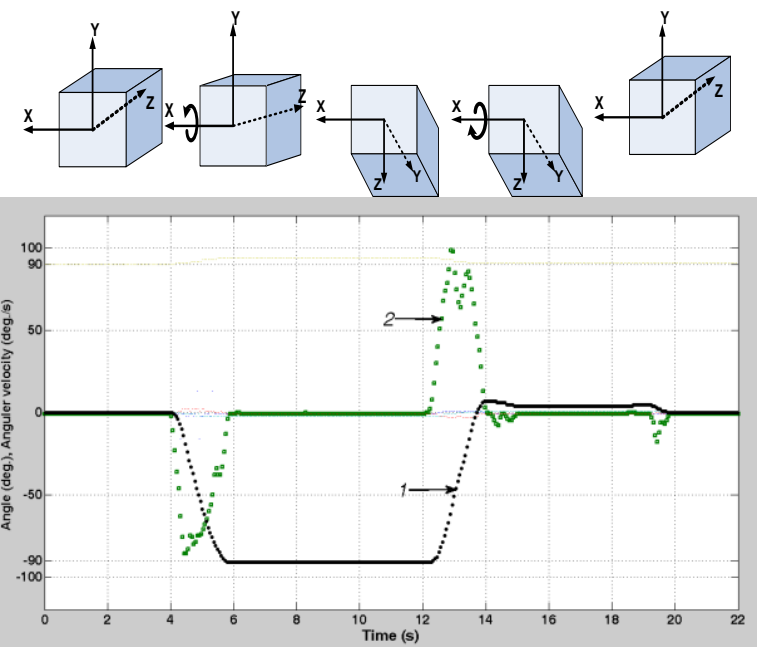

Fig. 7. Experiment results when rotating the object in space; 1 - estimation angles about $\mathrm{X}$ axis; 2 - estimation angular velocities about $\mathrm{X}$ axis.

After several experiments, the estimation angle static accuracy is statistically to be about 0.1 degrees. The update rate of $100 \mathrm{~Hz}$ can be achieved.

\section{CONCLUSIONS}

The experiment results show that the designed extended Kalman filter implementing in the dsPIC microcontroller guarantees real-time computation, the Kalman algorithm produces accurate angular estimation eliminating angular velocity drifts. A high speed DSP can be used to increase the update rate. The designed module is low cost and suitable for object attitude estimation in practical embedded applications.

\section{REFERENCES}

[1] J. L. Crassidis, F. L. Markley, and Y. Cheng, "Survey of nonlinear attitude estimation methods," Journal of Guidance Control and Dynamics, 2007.

[2] P. Batista, C. Silvestre, P. Oliveira, and B. Cardeira., "Low-cost attitude and heading reference system: Filter design and experimental evaluation," in Proc. IEEE International Conference on Robotics and Automation, May 2010, pp. 2624-2629.

[3] D. Gebre-Egziabher and G. Elkaim, "Mav attitude determination by vector matching," IEEE Transactions on Aerospace and Electronic Systems, vol. 44, no. 3, pp. 1012-1028, July 2008.

[4] H. Chao, C. Coopmans, L. Di, and Y. Q. Chen, "A comparative evaluation of low-cost IMUs for unmanned autonomous systems," in Proc. IEEE Conference on Multisensor Fusion and Integration for Intelligent System, University of Utah, Salt Lake City, Utah, USA September 2010, pp. 211-216.

[5] M. S. Grewal and A. P. Andrews, Kalman Filtering: Theory and Practice Using MATLAB. Wiley-Interscience, January 2001.

[6] R. E. Kalman, "A new approach to linear Filtering and prediction problems," Transactions of the ASME Journal of Basic Engineering, no. 82, pp. 35-45, 1960.

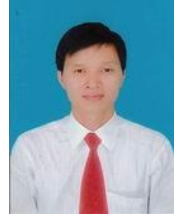

Bui Hong Hue received the Master of Engineering degree from the Military Technical Academy, Hanoi, Vietnam in 2006. He is presently the director of the College of Urban Works Construction, Hanoi, Vietnam. His current research interests include measurement and automatic control, embedded systems, DSP. In these areas, he has published 2 papers and 1 monograph. 


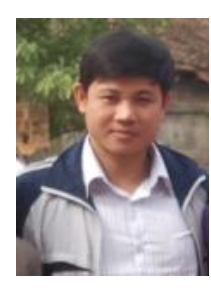

Tran Xuan Kien received the Ph.D. degree from the Saint Peterburg State Electrotechnical University, Russia in 2006. He is presently a researcher in the Military Science and Technology Institute, Hanoi, Vietnam. His current research interests include measurement and automatic control, embedded systems, DSP. In these areas, he has published more than 20 papers and 2 monographs and been awarded several research grants since 2006 .

Doan Minh Dinh received the Master of Engineering degree in Telecommunications from the Asian Institute of Technology in 2000 and the Ph.D. degree from the Military Technical Academy, Hanoi, Vietnam in 2005.

$\mathrm{He}$ is presently the head of the Deparment of Science and Technology, Ministry of Defense, Vietnam. His current research interests include automatic control, wireless communications, embedded systems.
Do Duc Hanh received the Master of Engineering degree in Electronic Engineering from the Military Technical Academy, Hanoi, Vietnam in 1992, the Master of Engineering and Ph.D. degrees in Computer Science from the Asian Institute of Technology in 1995 and 2010 respectively.

$\mathrm{He}$ is presently a lecturer of the Military Technical Academy, Hanoi, Vietnam. His current research interests include logic programming, augmentation, multiagent systems, automatic control, embedded systems, robotics and DSP. 